\title{
Quantum Theory of Surface Plasmon Polaritons: Planar and Spherical Geometries
}

\author{
Filippo Alpeggiani • Lucio Claudio Andreani
}

Received: 12 December 2013 / Accepted: 27 February 2014 / Published online: 3 April 2014

(C) Springer Science+Business Media New York 2014

\begin{abstract}
A quantum theory of retarded surface plasmons on a metal-vacuum interface is formulated, by analogy with the well-known and widely exploited theory of exciton-polaritons. The Hamiltonian for mutually interacting instantaneous surface plasmons and transverse electromagnetic modes is diagonalized with recourse to a Hopfield-Bogoljubov transformation, in order to obtain a new family of modes, to be identified with retarded plasmons. The interaction with nearby dipolar emitters is treated with a full quantum formalism based on a general definition of modal effective volumes. The illustrative cases of a planar surface and of a spherical nanoparticle are considered in detail. In the ideal situation of absence of dissipation, as an effect of the conservation of in-plane wavevector, retarded plasmons on a planar surface represent true stationary states (which are usually called surface plasmon polaritons), whereas retarded plasmons in a spherical nanoparticle, characterized by frequencies that overlap with the transverse electromagnetic continuum, become resonances with a finite radiative broadening. The theory presented constitutes a suitable full quantum framework for the study of nonperturbative and nonlinear effects in plasmonic nanosystems.
\end{abstract}

Keywords Quantum plasmonics · Surface plasmon polaritons $\cdot$ Radiative broadening

F. Alpeggiani $(\bowtie) \cdot$ L. C. Andreani

Dipartimento di Fisica, Università degli Studi di Pavia,

Via Bassi 6, 27100 Pavia, Italy

e-mail: filippo.alpeggiani01@ateneopv.it

\section{Introduction}

Plasmonic excitations on metal-dielectric interfaces are subject of large investigation from both the theoretical and the experimental points of view, because they could provide nanoscale confinement of light, and, consequently, they constitute a framework in which radiation-matter interaction phenomena are strongly enhanced [1]. In recent years, quantum phenomena associated to surface plasmons constitute a new focus within the name of quantum plasmonics. Unbounded metal-dielectric interfaces, such as planar surfaces, are characterized by propagating plasmonic excitations [2, 3], whereas confined nanostructures, like metallic particles, possess a discrete spectrum of surface plasmons. In the case of metallic spheres, the optical response can be calculated analytically with the well-known Mie theory [4-6]. In more complex geometries, the properties of plasmonic excitations can be studied analytically in the quasistatic approximation (i.e., neglecting retardation effects), which is valid when the characteristic size of the system is smaller than the wavelength of light, or with numerical methods, such as the boundary element method [7-9], the discrete dipole approximation $[10,11]$, or the finitedifference time-domain method [12].

The excitation of surface plasmons leads to a strong enhancement of radiation-matter interaction, which should be treated in the natural context of quantum electrodynamics (QED). A general quantization procedure for inhomogeneous systems is to model the electromagnetic response of the different media with a fictitious set of spatially distributed harmonic oscillators [13-16], whose amplitudes are related to the electromagnetic field through the dyadic Green function. The procedure is suitable to all kinds of dispersive and dissipative systems, including plasmonic systems, since the electromagnetic properties of the media are 
taken into account through the macroscopic dielectric function. This approach can be employed to study nonperturbative effects in the emission spectrum of quantum emitters coupled to surface plasmons, such as vacuum Rabi splitting $[17,18]$ or the onset of a Mollow triplet [19]. A different procedure is to directly quantize the eigenmodes of the electromagnetic field by associating them to a proper family of bosonic operators. This approach has been applied, for instance, to the specific case of a planar interface [20-25] and to the context of the surface plasmon amplification by stimulated emission of radiation (SPASER) theory [26] in the electrostatic approximation. Interestingly, light-matter interaction can be described with the well-established formalism of cavity QED, with surface plasmons playing the role of cavity modes.

A typical QED phenomenon taking place in confined dielectric systems is the Purcell effect, i.e., the modification of the decay rate of a quantum emitter due to the excitation of localized electromagnetic modes. The strength of the effect is proportional to the $Q$-factor of the excited mode and inversely proportional to its effective volume. The effective volume should be regarded as an important property of surface plasmons. In typical cavity QED systems, such as microcavities, modal effective volumes are subject to the diffraction limit, and they are at least of the order of $\lambda^{3}$, where $\lambda$ is the wavelength of light. As a consequence, enhancement of light-matter interaction is achieved by increasing the cavity $Q$-factor (for instance, by reducing radiative losses). In plasmonic systems, on the other hand, the situation is reversed. The width of plasmonic modes (and, consequently, the $Q$-factor) is limited by dissipation effects inside the metal, and it is generally of the order of the Drude damping constant $\gamma_{D}$. However, radiation being localized at a subwavelength scale, modal effective volumes can be made much smaller than the diffraction limit, leading to an enhancement of light-matter coupling often comparable to dielectric systems.

While the modal effective volume has a generally agreed definition for dielectric systems [27], a similar treatment for metallic systems is more complex, since they are inherently dispersive and dissipative. Recent solutions to the problem involve a reformulation of the definition of effective volumes [28] or a generalization of the Purcell equation [29]. In this work, we follow a different approach. We begin from the quantization of instantaneous surface plasmons, for which it is possible to introduce a rigorous definition of effective volumes, as suggested by Refs. [30-32]. This definition can be directly employed in a cavity QED formalism for treating, e.g., spontaneous emission modification.

Then, we include the effect of radiation and retardation with a procedure inspired by the theory of excitonpolaritons [33-36], based on a Hopfield-Bogoljubov transformation $[34,37]$ of the instantaneous plasmon operators.
Many results that are known from the theory of excitonpolaritons can be translated to surface plasmon polaritons, in particular, those related to the new vacuum state of coupled excitations, which does not coincide with the uncoupled vacuum [38]. Moreover, the present formalism clarifies the distinction between stationary surface plasmon polaritons in extended geometry and radiative surface plasmons in confined geometry. While we exemplify this distinction for the cases of planar and spherical surface, the general formalism can be applied to any specific situation.

The paper is organized as follows. In the "Quantization of Instantaneous Surface Plasmons" section, we introduce a general quantization procedure for instantaneous surface plasmons of a confined electron gas. Then, in the "Interaction with Matter: Effective Volumes" section, we define the effective volume for instantaneous excitations and treat the interaction with dipolar emitters by means of a cavity QED-inspired formalism based on the Purcell equation. The theory of the Hopfield-Bogoljubov transformation to include retardation effects is introduced in the "Retarded Surface Plasmons" section. As two illustrative cases, in the "Planar Geometry" and "Spherical Geometry" sections, a planar metal-vacuum interface and a spherical nanoparticle are considered, respectively, and the Hopfield-Bogoljubov transformation is carried out both analytically ("Planar Geometry" section) and numerically ("Spherical Geometry" section). The properties of retarded surface modes obtained in this way are analyzed, especially with reference to light-matter interaction. Finally, some concluding remarks are reported in the "Conclusions." Appendix A and Appendix B contain some intermediate results for the calculations presented in the "Planar Geometry" and "Spherical Geometry" sections, respectively.

\section{General Quantum Formulation}

\section{Quantization of Instantaneous Surface Plasmons}

In this work, we consider a (possibly unbounded) metallic region of space $\mathscr{M}$, in which a free electron gas is confined. As a consequence of electronic motion, in region $\mathscr{M}$, there is an average polarization density $\mathbf{P}(\mathbf{r})=-e n \mathbf{x}(\mathbf{r})$, where $\mathbf{x}(\mathbf{r})$ is the displacement field of the electrons and $n$ the electronic density. Electronic motion, in turn, gives rise to charge inhomogeneity and induces an electrostatic field $\mathbf{E}_{\mathrm{qs}}$, which is related to the polarization field by the classical equation of motion

$\ddot{\mathbf{P}}=-n e \ddot{\mathbf{x}}=\frac{n e^{2}}{m_{e}} \mathbf{E}_{\mathrm{qs}}=\varepsilon_{0} \omega_{\mathrm{P}}^{2} \mathbf{E}_{\mathrm{qs}}$, 
where we have introduced the plasma frequency $\omega_{\mathrm{P}}$. Instantaneous or quasistatic surface plasmons constitute an orthogonal basis $\phi_{n}$ for the electrostatic potential satisfying the Laplace equation $\nabla^{2} \phi_{n}=0$ in the different regions of space. The dispersion relation, i.e., the relation between the characteristic frequency of each mode $\omega_{n}$ and the mode index $n$, is obtained from the continuity conditions for the electric and displacement fields on the surface [39].

The Hamiltonian for the system consists of two contributions: the kinetic energy of the electrons and the potential energy due to the surface charge density:

$H_{\mathrm{qs}}=\int \mathrm{d}^{3} \mathbf{r}\left[\frac{\dot{P}^{2}(\mathbf{r})}{2 \varepsilon_{0} \omega_{\mathrm{P}}^{2}} \chi(\mathbf{r})+\frac{\varepsilon_{0}}{2} E_{\mathrm{qs}}^{2}(\mathbf{r})\right]$

$[\chi(\mathbf{r})$ is the characteristic function of the region $\mathscr{M}]$. By introducing the bosonic operators $\mathrm{b}_{n}$ and $\mathrm{b}_{n}^{\dagger}$, which satisfy the commutation relations ${ }^{1}$

$\left[\mathrm{b}_{n}, \mathrm{~b}_{n^{\prime}}^{\dagger}\right]=\delta_{n n^{\prime}} \quad$ and $\quad\left[\mathrm{b}_{n}, \mathrm{~b}_{n^{\prime}}\right]=\left[\mathrm{b}_{n}^{\dagger}, \mathrm{b}_{n^{\prime}}^{\dagger}\right]=0$,

the electric and polarization fields can be written as quantum operators expanded onto the family of electrostatic modes $\mathbf{E}_{\mathrm{qs}, n}=-\nabla \phi_{n}$, in the following form:

$$
\begin{aligned}
\mathrm{E}_{\mathrm{qs}}(\mathbf{r}) & =\sum_{n} \frac{1}{E_{n}} \sqrt{\frac{\hbar \omega_{n}}{2 \varepsilon_{0} V_{n}}} \\
& \times\left(\mathbf{E}_{\mathrm{qs}, n}(\mathbf{r}) \mathrm{b}_{n}+\mathbf{E}_{\mathrm{qs}, n}^{*}(\mathbf{r}) \mathrm{b}_{n}^{\dagger}\right) ; \\
\dot{\mathbf{P}}(\mathbf{r})= & i \varepsilon_{0} \omega_{\mathrm{P}}^{2} \chi(\mathbf{r}) \sum_{n} \frac{1}{E_{n}} \sqrt{\frac{\hbar}{2 \omega_{n} \varepsilon_{0} V_{n}}} \\
\times & \left(\mathbf{E}_{\mathrm{qs}, n}(\mathbf{r}) \mathrm{b}_{n}-\mathbf{E}_{\mathrm{qs}, n}^{*}(\mathbf{r}) \mathrm{b}_{n}^{\dagger}\right)
\end{aligned}
$$

(the $E_{n}$ 's are normalization constants). Then, the Hamiltonian is reduced to the harmonic form

$H_{\mathrm{qs}}=\sum_{n} \hbar \omega_{n}\left(\mathrm{~b}_{n}^{\dagger} \mathrm{b}_{n}+\frac{1}{2}\right)$,

provided that the modal volume $V_{n}$ is defined through the expression

$V_{n} \delta_{n n^{\prime}}=\frac{1}{2 E_{n}^{2}} \int \mathrm{d}^{3} \mathbf{r}\left(1+\frac{\omega_{\mathrm{P}}^{2}}{\omega_{n}^{2}} \chi(\mathbf{r})\right) \mathbf{E}_{\mathrm{qs}, n}^{*}(\mathbf{r}) \cdot \mathbf{E}_{\mathrm{qs}, n^{\prime}}(\mathbf{r})$.

This equation can be directly generalized by analogy with the formula for the electromagnetic energy of

\footnotetext{
${ }^{1}$ We notice that volume and surface plasmons are collective excitations that are formed in the subspace of electron-hole pair excitations, which have integer spin and, therefore, bosonic character. Bosonic commutation relation is obeyed in the limit of weak excitation, while corrections are expected to be of the order of $P / V$, where $P$ is the number of excited plasmons and $V$, the crystal volume. The situation is analogous to the case of exciton states, as discussed in Hopfield's seminal work (Ref. [34]).
}

a dispersive system [40] and recast into the following form:

$$
V_{n} \delta_{n n^{\prime}}=\frac{1}{2 E_{n}^{2}} \int \mathrm{d}^{3} \mathbf{r} \frac{\partial[\omega \Re \varepsilon(\omega)]}{\partial \omega} \mathbf{E}_{\mathrm{qs}, n}^{*}(\mathbf{r}) \cdot \mathbf{E}_{\mathrm{qs}, n^{\prime}}(\mathbf{r}) .
$$

Notice, in particular, that the general expression (7) reduces to Eq. 6 when the Drude dielectric function for the free electron gas is used. In this work, we have supposed that the macroscopic response of the electron gas can be modeled with a local dielectric function; the effect of spatial confinement on the relaxation rate $\gamma_{D}$ [41] and the electronic eigenstates of the metal [42] is neglected. This is a good approximation as long as the size of the nanostructure is larger than the so-called nonlocality length (about $1 \mathrm{~nm}$ in the optical region [1]).

\section{Interaction with Matter: Effective Volumes}

At this point, we suppose to add a single dipolar emitter (atom, molecule, quantum dot, etc.) in the region of space outside $\mathscr{M}$. As an effect of the strong localization of the electric field at the boundary of $\mathscr{M}$, the spontaneous emission rate of the atom can be significantly modified with respect to free-space (Purcell effect).

In the electrostatic approximation, the Hamiltonian of the total system (electron gas + atom) can be written in the following form: $H=H_{\mathrm{qs}}+H_{\mathrm{a}}+H_{\mathrm{int}}$, where $H_{\mathrm{qs}}$ is defined in Eq. $2, H_{\mathrm{a}}$ is the unperturbed atomic Hamiltonian, and $H_{\text {int }}$ is the electrostatic energy of the atomic charges in the external potential generated by the free electron gas. The atom can be treated as a two-level system with ground state $|\mathrm{gr}\rangle$ and excited state $|\mathrm{ex}\rangle$. In the dipole approximation, $H_{\text {int }}$ can be written as follows:

$H_{\text {int }}=-\mu\left(\sigma_{-}+\sigma_{+}\right) \cdot \mathrm{E}_{\mathrm{qs}}\left(\mathbf{r}_{\mathrm{a}}\right)$.

( $\sigma_{ \pm}$are the Pauli operators and $\mathbf{r}_{\mathrm{a}}$ is the atomic center of mass position).

According to perturbation theory, the decay rate of the atom into plasmonic modes is calculated by means of the Fermi golden rule:

$\Gamma_{\mathrm{qs}}=\frac{2 \pi}{\hbar^{2}} \sum_{n}\left|\left\langle\mathrm{gr}, 1_{n}\left|\mu \cdot \mathrm{E}_{\mathrm{qs}}\left(\mathbf{r}_{\mathrm{a}}\right) \sigma_{-}\right| \mathrm{ex}, 0\right\rangle\right|^{2} \delta\left(\omega_{n}-\omega_{\mathrm{a}}\right)$.

In actual systems, resonances in the plasmonic density of states are broadened due to dissipation-induced damping of the electron motion in the gas (which we have so far neglected); this effect can be phenomenologically included in our treatment by replacing the delta functions in the Fermi golden rule with normalized Lorentzian functions $\gamma_{\mathrm{D}} /\left[2 \pi\left(\left(\omega-\omega_{\mathrm{a}}\right)^{2}+\gamma_{\mathrm{D}}^{2} / 4\right)\right]$, whose width $\gamma_{\mathrm{D}}$ can be directly identified with the damping constant that enters the complex 
Drude dielectric function $\varepsilon(\omega)=1-\omega_{\mathrm{P}}^{2} /\left[\omega\left(\omega+i \gamma_{\mathrm{D}}\right)\right]$. As a result, the decay rate becomes

$\Gamma_{\mathrm{qs}}=\frac{\mu^{2}}{\hbar} \sum_{n} \frac{\omega_{n}\left|\hat{\mu} \cdot \mathbf{E}_{\mathrm{qs}, n}\left(\mathbf{r}_{\mathrm{a}}\right)\right|^{2}}{2 \varepsilon_{0} V_{n} E_{n}^{2}} \frac{\gamma_{\mathrm{D}}}{\left(\omega_{n}-\omega_{\mathrm{a}}\right)^{2}+\gamma_{\mathrm{D}}^{2} / 4}$,

where $\hat{\mu}$ is the unit vector directed as $\mu$.

Equation 10 can be recast in a simpler form by taking the arbitrary constant $E_{n}$ equal to $\left|\hat{\mu} \cdot \mathbf{E}_{\mathrm{qs}, n}\left(\mathbf{r}_{\mathrm{a}}\right)\right|$ in Eq. 7; as a consequence, we are led to the definition of the spatiallydependent effective volume

$$
\begin{aligned}
& V_{n}\left(\mathbf{r}_{\mathrm{a}}, \hat{\mu}\right) \delta_{n n^{\prime}}=\frac{1}{2\left|\hat{\mu} \cdot \mathbf{E}_{\mathrm{qs}, n}\left(\mathbf{r}_{\mathrm{a}}\right)\right|^{2}} \times \\
& \quad \int \mathrm{d}^{3} \mathbf{r} \frac{\partial[\omega \Re \varepsilon(\mathbf{r}, \omega)]}{\partial \omega} \mathbf{E}_{\mathrm{qs}, n}^{*}(\mathbf{r}) \cdot \mathbf{E}_{\mathrm{qs}, n^{\prime}}(\mathbf{r}) .
\end{aligned}
$$

As it can be seen, the effective volume is one half of the volume of a hypothetical cavity containing the same energy as the plasmonic mode, with the condition for the field inside the cavity of being homogeneous and of the same magnitude as the field at the atom position. Equation 11 differs from the analogous definition of the effective volume in cavity QED [27] by the presence of a factor $1 / 2$ in the right-hand term since, in the range of validity of the quasistatic approximation, the magnetic contribution to the electromagnetic energy is absent. At variance from typical cavity QED systems, such as optical cavities, the relation $\mathbf{E} \cdot \mathbf{D}=\mathbf{H} \cdot \mathbf{B}$ does not hold for plasmonic systems in the quasistatic approximation due to the presence of evanescent electromagnetic waves. However, in the "Planar Geometry" section, we show that, when retardation effects become prevailing, the magnetic contribution becomes significant even for plasmonic systems.

With the new definition of the effective volume in Eq. 11, the decay rate (Eq. 10) assumes the form of the Purcell equation

$\Gamma_{\mathrm{qs}}=\Gamma_{0} \sum_{n} \frac{3 \lambda_{\mathrm{a}}^{3}}{4 \pi^{2}} \frac{Q_{n}}{V_{n}\left(\mathbf{r}_{\mathrm{a}}, \hat{\mu}\right)} \frac{\gamma_{\mathrm{D}}^{2} / 4}{\left(\omega_{n}-\omega_{\mathrm{a}}\right)^{2}+\gamma_{\mathrm{D}}^{2} / 4}$

where $\lambda_{\mathrm{a}}=2 \pi c / \omega_{\mathrm{a}}$, the $Q$-value of the plasmonic resonance is the ratio $Q_{n}=\omega_{n} / \gamma_{\mathrm{D}}$, and $\Gamma_{0}$ is the free-space decay rate $\Gamma_{0}=\omega_{\mathrm{a}}^{3} \mu^{2} /\left(3 \pi \varepsilon_{0} \hbar c^{3}\right)$.

The interest of Eqs. 11 and 12 resides in the fact that they allow to study radiation-matter interaction with a cavity QED-inspired formalism. Notice that the analogy with cavity QED is not limited to the perturbative decay rate. The electric field operator defined in Eq. 3 can be employed to generalize several cavity QED results in a straightforward manner. For instance, when light-matter interaction is significantly enhanced by the electric field confinement, the atom can enter the nonperturbative (strong-coupling) regime, characterized by the onset of a doublet of peaks around the transition frequency in the emission spectrum (vacuum Rabi splitting) [17, 43-47]. The condition for entering the nonperturbative regime via the coupling with a single plasmonic mode can be expressed by analogy with the cavity QED formalism in the form $g>\left|\gamma_{\mathrm{D}}-\gamma_{\mathrm{a}}\right| / 4$ [48], where $\gamma_{\mathrm{D}}$ and $\gamma_{\mathrm{a}}$ are the linewidths of the plasmonic mode and the atom, respectively, and the coupling constant $g$ is a function of the effective volume in Eq. 11 and the atomic oscillator strength $f$, according to the relation

$g=\frac{1}{2} \sqrt{\frac{e^{2} f}{m_{e} \varepsilon_{0} V_{n}\left(\mathbf{r}_{\mathrm{a}}, \hat{\mu}\right)}}$.

Therefore, effective volumes calculated with Eq. 11 can be employed to determine the threshold for entering the strongcoupling regime in a straightforward way. For instance, calculations for a metallic nanoshell based on Eq. 13 are presented in Ref. [49].

The results derived above are valid as long as retardation effects can be neglected, which is not the case for several regimes of great interest. However, retardation can be taken into account with a full quantum formalism based on the theory of exciton-polaritons, as it will be shown in the following.

\section{Retarded Surface Plasmons}

We turn the attention to the study of retardation effects, which become relevant with the increase of the characteristic size of the system. The quantum theory of retarded surface plasmons can be constructed by analogy with that of exciton-polaritons [33-36]: each instantaneous surface plasmon of the metallic surface (playing the role of the exciton) interacts with the quantized modes of the transverse electromagnetic field in vacuum, which are described by the vector potential $\mathbf{A}(\mathbf{r})$.

We choose to work in the Coulomb gauge, with $\nabla \cdot \mathbf{A}=0$ everywhere, including the boundary of $\mathscr{M}$. With this choice, instantaneous plasmons are fully described by the electrostatic potential $\phi$, whereas the transverse electromagnetic field is taken into account by means of the transverse vector potential A. Notice that this is at variance with other works on surface plasmons $[20,22,25]$ or intersubband polaritons [50], in which the dipole gauge ${ }^{2}$ is employed. The minimal coupling Hamiltonian for the system (excluding for the

\footnotetext{
${ }^{2}$ In the dipole gauge, the electrostatic potential $\phi$ is not used; the Coulomb interaction arouses from the longitudinal part of a $P^{2}$ term in the Hamiltonian. Light-matter coupling is included by means of the Power-Zienau transformation with a term of the form $-\mu \cdot \mathbf{E}$. In this work, however, we employ the Coulomb gauge in order to keep the instantaneous and transverse characters of the field separated. On the choice of the gauge, see also Refs. [22] and [50].
} 
moment the interaction with external atoms or molecules) can be written as follows:

$$
\begin{aligned}
H_{\mathrm{ret}} & =H_{\mathrm{qs}}+H_{\mathrm{elm}}+H_{\mathrm{I}}+H_{\mathrm{II}}, \\
H_{\mathrm{elm}} & =\int \mathrm{d}^{3} \mathbf{r}\left[\frac{\varepsilon_{0}}{2} \dot{A}^{2}+\frac{1}{2 \mu_{0}}(\nabla \times \mathbf{A})^{2}\right], \\
H_{\mathrm{I}} & =-\int \mathrm{d}^{3} \mathbf{r} \dot{\mathbf{P}}(\mathbf{r}) \cdot \mathbf{A}(\mathbf{r}), \\
H_{\mathrm{II}} & =\frac{\varepsilon_{0} \omega_{\mathrm{P}}^{2}}{2} \int \mathrm{d}^{3} \mathbf{r} \chi(\mathbf{r}) A^{2}(\mathbf{r}),
\end{aligned}
$$

and $H_{\mathrm{qs}}$ is defined in Eq. 5. Notice the term $H_{\mathrm{II}}$, which couples together different modes of the transverse field due to the spatial inhomogeneity induced by the presence of the region $\mathscr{M}$ of free electron gas.

The transverse vector potential can be quantized upon expansion onto a continuum of transverse electromagnetic modes $\mathbf{A}_{\mathrm{T}, v}(\mathbf{r})$ (e.g., plane waves), each labeled by a continuous index $v$ and with energy $\omega_{\nu}$, by introducing a family of bosonic operators $\mathrm{a}_{v}$ and $\mathrm{a}_{v}^{\dagger}$ :

$\mathrm{A}(\mathbf{r})=\sum_{v}\left[\mathbf{A}_{\mathrm{T}, v}(\mathbf{r}) \mathrm{a}_{v}+\mathbf{A}_{\mathrm{T}, v}^{*}(\mathbf{r}) \mathrm{a}_{v}^{\dagger}\right]$.

With the aid of Eq. 4, the Hamiltonian can be expressed in the following form :

$$
\begin{aligned}
H_{\text {elm }} & =\sum_{v} \hbar \omega_{v}\left(\mathrm{a}_{v}^{\dagger} \mathrm{a}_{v}+\frac{1}{2}\right) ; \\
H_{\mathrm{I}} & =i \sum_{n, v} \hbar C_{n, v}\left(\mathrm{~b}_{n}-\mathrm{b}_{n}^{\dagger}\right)\left(\mathrm{a}_{v}+\mathrm{a}_{v}^{\dagger}\right) ; \\
H_{\mathrm{II}} & =\sum_{v, v^{\prime}} \hbar D_{v, v^{\prime}}\left(\mathrm{a}_{v}+\mathrm{a}_{v}^{\dagger}\right)\left(\mathrm{a}_{v^{\prime}}+\mathrm{a}_{v^{\prime}}^{\dagger}\right),
\end{aligned}
$$

$C$ and $D$ are coefficients depending on the particular geometry, as shown in Appendices A and B. Interaction terms in Eqs. 20-21 are analogous to those of exciton-polaritons [34].

For each instantaneous plasmonic mode $n$, the Hamiltonian can be diagonalized with a Hopfield-Bogoljubov transformation by introducing a new family of operators $\alpha_{n}$ as linear combinations of the unperturbed ones:

$\alpha_{n}=W_{n} \mathrm{~b}_{n}+Y_{n} \mathrm{~b}_{n}^{\dagger}+\sum_{\nu}\left(X_{n, v} \mathrm{a}_{v}+Z_{n, v} \mathrm{a}_{v}^{\dagger}\right)$.

The harmonic condition

$\left[\alpha_{n}, H_{\text {ret }}\right]=\hbar \Omega_{n} \alpha_{n}$

assumes the form of an eigenvalue equation which provides the solutions for the coefficients $W_{n}, Y_{n}, X_{n, v}, Z_{n, v}$, and the modified eigenfrequencies $\Omega_{n}$ of the retarded modes.

Except for the additional term $H_{\mathrm{II}}$, the Hamiltonian in Eq. 14 coincides with that of the Fano-Anderson model of a discrete state in interaction with a continuum [51, 52], which gives rise to two distinct physical situations. If the retarded mode frequency $\Omega_{n}$ does not overlap with the spectrum of transverse modes $\omega_{\nu}$, the corresponding operator $\alpha_{n}$ represents a true stationary state of the system; an example is provided by the surface plasmon polariton at a planar metal-vacuum interface. On the other hand, when the frequency $\Omega_{n}$ lies in the $\omega_{\nu}$ spectrum, the instantaneous plasmon becomes a scattering resonance in the continuum of electromagnetic modes. In this case, we can introduce the density of states of the quasistatic mode (called admixture density in Ref. [51]), defined as

$\rho(\Omega)=\sum_{j}\left[\left|W_{n}^{(j)}\right|^{2} \delta\left(\Omega-\Omega_{n}^{(j)}\right)\right]$,

where the index $(j)$ identifies the different eigenvalues of Eq. 23. As an effect of the interaction with the continuum of electromagnetic modes, this quantity assumes a finite linewidth. The phenomenon is commonly addressed as radiative broadening of surface plasmons, and it is characteristic of a fully confined metal nanostructure, such as a metal nanosphere. We stress that radiative broadening is not a dissipation effect, and it is present even in ideal nondissipative systems, such as those considered in this work.

In the following sections, we will work out the calculations and analyze the properties of retarded plasmons for both the planar interface and the spherical geometry, discussing in particular light-matter coupling between the plasmonic excitations and a nearby dipolar emitter.

\section{Planar Geometry}

Instantaneous Modes

In this section, we suppose the region $\mathscr{M}$ to be the halfspace defined by the condition $z<0$. By solving the Laplace equation with the proper boundary conditions at the interface $z=0$, we find a continuous set of instantaneous modes for the electrostatic field, indexed by the in-plane wavevector $\mathbf{k}_{\|}$

$\mathbf{E}_{\mathrm{qs}, \mathbf{k}_{\|}}(\mathbf{r})=-\left(i \mathbf{k}_{\|} \mp k_{\|} \hat{\mathbf{z}}\right) e^{i \mathbf{k}_{\|} \cdot \rho-k_{\|}|z|}$

(the upper and lower signs refer to the regions $z>0$ and $z<$ 0 , respectively, and $\rho=x \hat{\mathbf{x}}+y \hat{\mathbf{y}})$. All instantaneous modes are characterized by the same frequency $\omega_{\mathrm{s}}=\omega_{\mathrm{P}} / \sqrt{2}$. We suppose that an atom is located at a distance $z_{\mathrm{a}}$ above the surface, and the dipole moment is oriented perpendicular to it (along $\hat{\mathbf{z}}$ ). In the quasistatic approximation, it is possible to define the effective volume $V_{n}$ according to Eq. 11 . For the planar geometry, being the index $\mathbf{k}_{\|}$continuous, the effective volume becomes the effective volume density

$\mathscr{V}_{\mathbf{k}_{\|}, \mathrm{qs}}\left(z_{\mathrm{a}}, \hat{\mathbf{z}}\right)=8 \pi^{2} e^{2 k_{\|} z_{\mathrm{a}}} / k_{\|}$. 
The density has the dimensions of a volume per unit surface, i.e., it can be equally interpreted as an effective length [30], which provides an estimate of the confinement of the field along the direction perpendicular to the surface. Such effective length, in the $z_{\mathrm{a}}=0$ case, is of the order of the free-space wavelength, in agreement with the analogous behavior of the penetration depth of the field into the dielectric [53], which is the dominant term for the spatial localization of surface plasmons. Replacing $\mathscr{V}_{\mathbf{k}}$, qs into the Purcell Eq. 12, we obtain the decay rate in the quasistatic approximation $\left(k_{\mathrm{a}}=\omega_{\mathrm{a}} / c\right)$

$$
\begin{aligned}
\frac{\Gamma_{\mathrm{qs}}}{\Gamma_{0}} & =\frac{3 \lambda_{\mathrm{a}}^{3}}{4 \pi^{2}} \int \mathrm{d}^{2} \mathbf{k}_{\|} \frac{1}{\mathscr{y}_{\mathbf{k}_{\|}, \mathrm{qs}}}\left(\frac{\omega_{\mathrm{s}}}{\gamma_{\mathrm{D}}}\right) \frac{\gamma_{\mathrm{D}}^{2} / 4}{\left(\omega_{\mathrm{s}}-\omega_{\mathrm{a}}\right)^{2}+\gamma_{\mathrm{D}}^{2} / 4} \\
& =\frac{3 \omega_{\mathrm{s}}}{8 \gamma_{\mathrm{D}}\left(k_{\mathrm{a}} z_{\mathrm{a}}\right)^{3}} \frac{\gamma_{\mathrm{D}}^{2} / 4}{\left(\omega_{\mathrm{s}}-\omega_{\mathrm{a}}\right)^{2}+\gamma_{\mathrm{D}}^{2} / 4} .
\end{aligned}
$$

This coincides with the quasistatic contribution which in Ref. [2] (Eq. 3.23) is attributed to lossy surface waves (i.e., instantaneous surface excitations), calculated for a complex Drude dielectric function and in the limit $\omega_{\mathrm{a}} \simeq \omega_{\mathrm{s}}$. It represents the dominant term when the surface-atom distance is small compared to the wavelength of light.

\section{Hopfield-Bogoljubov Transformation}

In order to treat retardation effects with the HopfieldBogoljubov transformation described in the "Retarded Surface Plasmons" section, the free-space vector potential has to be expanded onto a proper family of modes:

$\mathrm{A}=\int \mathrm{d}^{2} \mathbf{k}_{\|} \mathrm{d} k_{z} \mathbf{A}_{\mathrm{T}, \mathbf{k}_{\|}, k_{z}}\left[\mathrm{a}_{\mathbf{k}_{\|}, k_{z}}+\mathrm{a}_{-\mathbf{k}_{\|},-k_{z}}^{\dagger}\right]$.

We choose free-space plane waves with wavevector $\mathbf{k}=$ $\mathbf{k}_{\|}+\hat{\mathbf{z}} k_{z}$ and frequency $\omega_{\mathbf{k}}=c k$, in the form

$\mathbf{A}_{\mathrm{T}, \mathbf{k}_{\|}, k_{z}}(\mathbf{r})=\sqrt{\frac{\hbar}{16 \pi^{3} c k \varepsilon_{0}}} \mathscr{E}_{\mathbf{k}_{\|}, k_{z}} e^{i \mathbf{k} \cdot \mathbf{r}}$,

with the polarization vector characteristic of transverse magnetic (TM) modes

$\mathscr{E}_{\mathbf{k}_{\|}, k_{z}}=\frac{1}{k k_{\|}}\left(k_{x} k_{z}, \quad k_{y} k_{z}, \quad-k_{\|}^{2}\right)^{T}$.

Transverse electric (TE) modes do not interact with the instantaneous plasmon, since $\dot{\mathbf{P}}(\mathbf{r}) \cdot \mathbf{A}(\mathbf{r})=0$.

The expressions for the terms $H_{\mathrm{I}}$ and $H_{\mathrm{II}}$ in the total Hamiltonian of Eq. 14 can be calculated analytically and are reported in Appendix A. Following the approach in the "Retarded Surface Plasmons" section, we diagonalize the Hamiltonian by introducing the new family of bosonic operators:

$$
\begin{aligned}
& \alpha_{\mathbf{k}_{\|}}=W \mathrm{~b}_{\mathbf{k}_{\|}}+Y \mathrm{~b}_{-\mathbf{k}_{\|}}^{\dagger}+ \\
& \int \mathrm{d} k_{z}\left[X\left(k_{z}\right) \mathbf{a}_{\mathbf{k}_{\|}, k_{z}}+Z\left(k_{z}\right) \mathrm{a}_{-\mathbf{k}_{\|},-k_{z}}^{\dagger}\right] .
\end{aligned}
$$

The condition (23) gives rise to an eigenproblem for the coefficients $W, X\left(k_{z}\right), Y, Z\left(k_{z}\right)$, which is solved in the complex $k_{z}$ plane with the procedure sketched in Appendix A. In particular, the solution is the well-known dispersion relation for surface plasmon polaritons [3], shown in Fig. 1:

$\Omega_{\mathbf{k}_{\|}}^{2} / \omega_{\mathrm{s}}^{2}=1+2\left(\frac{c k_{\|}}{\omega_{\mathrm{P}}}\right)^{2}-\sqrt{1+4\left(\frac{c k_{\|}}{\omega_{\mathrm{P}}}\right)^{4}}$.

In Fig. 1, in addition to $\Omega_{\mathbf{k}_{\|}}$, the coefficients $W$ and $Y$ are plotted as a function of the in-plane wavevector. In the limit $k_{\|} \rightarrow \infty, W$ tends to unity, whereas $Y$ tends to zero, indicating that the surface plasmon polariton reduces to the instantaneous plasmon, with the associated operator $b_{\mathbf{k}_{\|}}$. In the same limit, the retarded frequency $\Omega_{\mathbf{k}_{\|}}$tends to the instantaneous value $\omega_{\mathrm{s}}$.

The calculated dispersion relation corresponds to that obtained from classical electrodynamics, providing a confirmation of the validity of the theory. However, we believe that the results presented here have a broader range of application than a simple reformulation of classical electrodynamics, since we have derived an analytical expression for the quantum operators $\alpha_{\mathbf{k}_{\|}}$associated to surface plasmon polaritons, based on the photon and instantaneous plasmon operators. For instance, as it is characteristic of the Hopfield-Bogoljubov transformation [38], the polaritonic vacuum state $\left|0^{\prime}\right\rangle$, defined by the condition $\alpha_{\mathbf{k}_{\|}}\left|0^{\prime}\right\rangle=$ 0 , is different from the unperturbed vacuum state $|0\rangle$ of photons and instantaneous plasmons. This is evident from

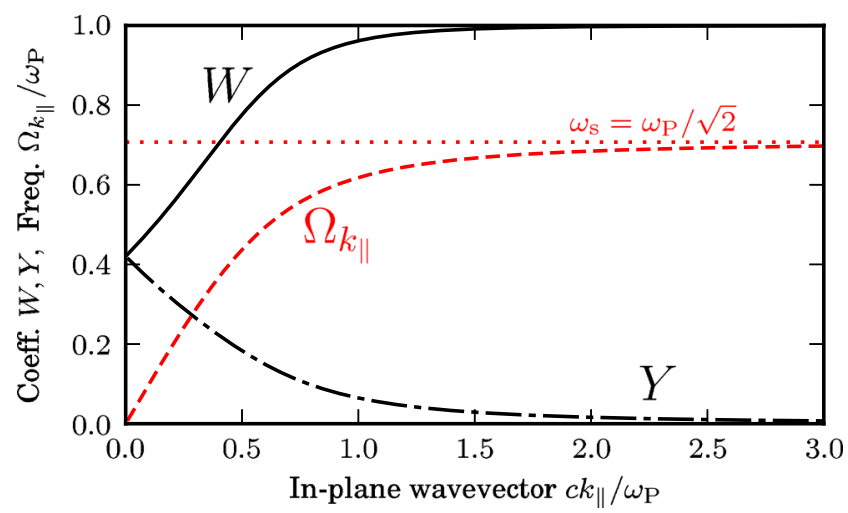

Fig. 1 The coefficients $W$ and $Y$ of the expansion in Eq. 29 and the frequency $\Omega_{k_{\|}}$of the surface plasmon polariton (normalized to $\omega_{\mathrm{P}}$ ), as a function of the in-plane wavevector $k_{\|}$(normalized to $\omega_{\mathrm{P}} / c$ ). The dotted line indicates the frequency $\omega_{\mathrm{s}}$ of the instantaneous surface plasmon 
the calculation of the average number of polaritons in the unperturbed vacuum

$\left\langle 0\left|\alpha_{\mathbf{k}_{\|}}^{\dagger} \alpha_{\mathbf{k}_{\|}}\right| 0\right\rangle=|Y|^{2}+\int \mathrm{d} k_{z}\left|Z\left(k_{z}\right)\right|^{2} \neq 0$,

a quantity which can be shown from the inversion relations of the Hopfield-Bogoljubov transformation [34] to be equivalent to the average number of instantaneous plasmons in the polaritonic vacuum $\left\langle 0^{\prime}\left|b_{\mathbf{k}_{\|}}^{\dagger} b_{\mathbf{k}_{\|}}\right| 0^{\prime}\right\rangle$. This quantity is plotted in Fig. 2 as a function of the in-plane wavevector. The deviation from the unperturbed vacuum state is maximum in the $k_{\|} \rightarrow 0$ limit, i.e., for the maximum coupling with radiation, where it tends to the value $\mathscr{K}^{2}=\omega_{\mathrm{s}} / 4 \omega_{\mathrm{P}}$ ( $\mathscr{K}$ is the normalization constant defined in Eq. 51). Notice that, being the curve a function of $c k_{\|} / \omega_{\mathrm{P}}$, for a fixed inplane wavelength, polaritonic effects can be enhanced by increasing the electronic density. Vacuum state modification as an effect of the interaction with light is a quantum phenomenon of great interest, especially in the context of the so-called ultrastrong coupling regime [54-56], which could be extended also to the framework of quantum plasmonics.

\section{Interaction with Matter}

In the context of the Hopfield-Bogoljubov transformation introduced above, it is easy to evaluate the effect of retardation onto the interaction between the plasmon and the atom at $z=z_{\mathrm{a}}$. The retarded electric field can be expanded onto the $\alpha_{\mathbf{k}_{\|}}$operators in the form

$\mathrm{E}=\int \mathrm{d}^{2} \mathbf{k}_{\|} \mathbf{E}_{\mathbf{k}_{\|}}\left(\alpha_{\mathbf{k}_{\|}}+\alpha_{-\mathbf{k}_{\|}}^{\dagger}\right)$

The expression for the modes $\mathbf{E}_{\mathbf{k}_{\|}}(\mathbf{r})$ is calculated in Appendix A, and it presents the same spatial dependence of the field calculated from classical electrodynamics.

By replacing the interaction term in Eq. 8 into the Fermi golden rule (9) and comparing it with Eq. 12, it is possible to

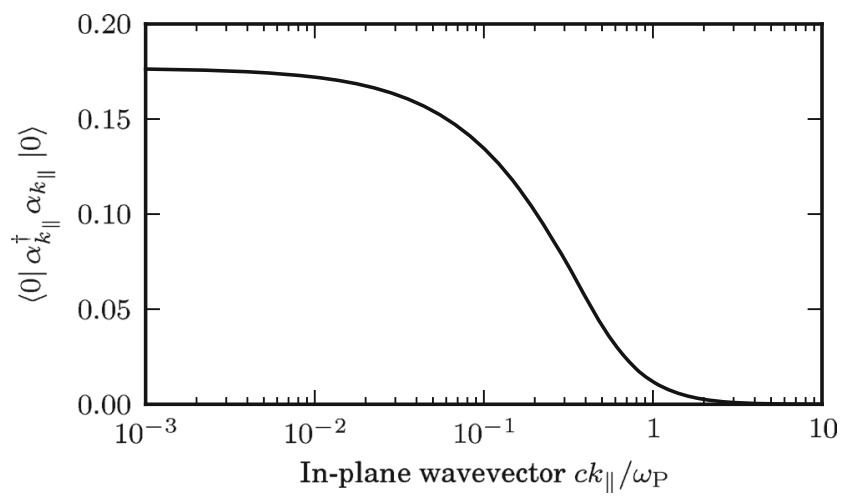

Fig. 2 The average number of surface plasmon polaritons in the unperturbed vacuum, as a function of the in-plane wavevector (normalized to $\omega_{\mathrm{P}} / c$ ) identify the effective volume density for retarded plasmon polaritons

$\mathscr{L}_{\mathbf{k}_{\|}}=\frac{2 \pi^{2}\left(\Delta_{+}+\Delta_{-}\right)\left(\Delta_{+}^{2}+\Delta_{-}^{2}\right)}{\left|\left(-i \hat{\mathbf{k}}_{\|}+\frac{k_{\|}}{\Delta_{+}} \hat{\mathbf{z}}\right) \cdot \hat{\mu}\right|^{2} k_{\|}^{4}} e^{2 z_{\mathbf{a}} \Delta_{+}}$

(the quantities $\Delta_{+}$and $\Delta_{-}$are defined in Appendix A). The reciprocal of the effective volume is represented in Fig. 3 as a function of the in-plane wavenumber $k_{\|}$, for several values of the atom-interface distance $z_{\mathrm{a}}$. In the Purcell formula (see Eq. 12), the reciprocal of $\mathscr{1} / \mathbf{k}_{\|}$could be interpreted as the weight of each modal contribution to the decay rate, so it basically represents a measure of the strength of radiationmatter interaction for a specific mode. As it can be seen in Fig. 3, the contribution from short-wavelength (high$\left.k_{\|}\right)$modes increases with the decrease of $z_{\mathrm{a}}$, i.e., when we approach the instantaneous limit with no significant retardation effects. This is in agreement with the behavior of the retarded frequency $\Omega_{\mathbf{k}_{\|}}$and the coefficients $W$ and $Y$ in Fig. 1.

The retarded effective volumes in Eq. 33 can be interpreted in a interesting manner, by observing that the definition of the effective volume in Eq. 11 can be generalized in the following form:

$$
\begin{aligned}
\mathscr{V}_{\mathbf{k}_{\|} \delta} \delta\left(\mathbf{k}_{\|}-\mathbf{k}_{\|}^{\prime}\right) & =\frac{\alpha\left(\mathbf{k}_{\|}\right)}{\left|\mathbf{E}_{\mathbf{k}_{\|}}\left(\mathbf{r}_{\mathrm{a}}\right) \cdot \hat{\mu}\right|^{2}} \times \\
& \int \mathrm{d}^{3} \mathbf{r} \frac{\partial[\omega \Re \varepsilon(\mathbf{r}, \omega)]}{\partial \omega} \mathbf{E}_{\mathbf{k}_{\|}}(\mathbf{r}) \cdot \mathbf{E}_{\mathbf{k}_{\|}^{\prime}}(\mathbf{r}) .
\end{aligned}
$$

The dependence of factor $\alpha\left(\mathbf{k}_{\|}\right)$on $\mathbf{k}_{\|}$-calculated by comparing Eq. 33 with the expression for the electric field $\mathbf{E}_{\mathbf{k}_{\|}}(\mathbf{r})$ reported in Appendix A-is plotted in Fig. 4. The presence of factor $\alpha\left(\mathbf{k}_{\|}\right)$must be taken into account in plasmonic systems since the energy stored in evanescent electric fields is not matched by a corresponding amount of magnetic energy. In purely dielectric systems, such as optical

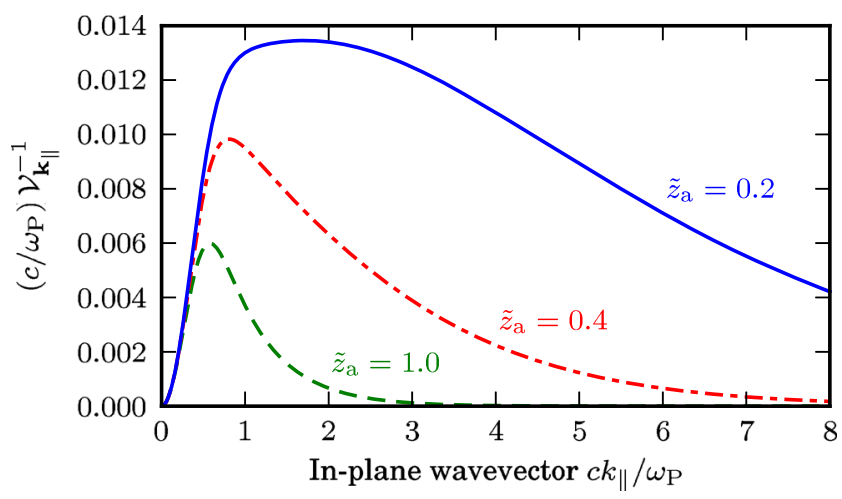

Fig. 3 The reciprocal of the effective volume density $\mathscr{V}_{\mathbf{k}_{\|}}$in Eq. 33 as a function of the in-plane wavenumber $k_{\|}$(both normalized to $\omega_{\mathrm{P}} / c$ ). Each curve is calculated for a different value of the (normalized) atominterface distance $\tilde{z}_{\mathrm{a}}=\omega_{\mathrm{P}} z_{\mathrm{a}} / c$, indicated by the label. The dipole at $z_{\mathrm{a}}$ is oriented as $\hat{\mathbf{z}}$ 


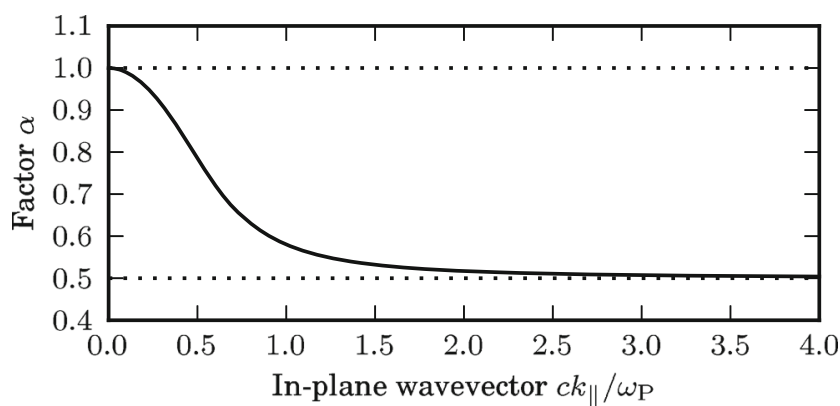

Fig. 4 The factor $\alpha\left(\mathbf{k}_{\|}\right)$(defined in Eq. 34) versus the normalized inplane wavevector. The two dotted lines indicate the instantaneous and retarded limits at $\alpha=1 / 2$ and $\alpha=1$, respectively

cavities [27], we expect the factor $\alpha\left(\mathbf{k}_{\|}\right)$to be unity, which is the $k_{\|} \rightarrow 0$ limit of the curve in Fig. 4. On the other hand, as we have already noted commenting Eq. 11, in the quasistatic regime, the value of $\alpha\left(\mathbf{k}_{\|}\right)$is exactly one half, in agreement with the large $k_{\|}$behavior of Fig. 4. Correspondingly, when the atom is very near the metal-dielectric interface, the magnetic contribution is negligible. As the atom is separated from the interface, however, there is a transition towards the retarded regime, and the magnetic energy contribution progressively increases, until it reaches the same magnitude as the electric one. At the same time, the effective volume is affected by the diffraction limit and progressively decreases, as proved by Fig. 3 .

The effective volume reported in Eq. 33 allows to calculate the modified decay rate of an atom into the retarded surface plasmon polaritons of a planar metal-vacuum interface with the Purcell equation

$\frac{\Gamma_{\mathrm{qs}}}{\Gamma_{0}}=\int \mathrm{d}^{2} \mathbf{k}_{\|} \frac{3 \lambda_{\mathrm{a}}^{3}}{4 \pi^{2}} \frac{Q_{\mathbf{k}_{\|}}}{\mathscr{V}_{\mathbf{k}_{\|}}} \frac{\gamma_{\mathbf{k}_{\|}}^{2} / 4}{\left(\omega_{\mathbf{k}_{\|}}-\omega_{\mathrm{a}}\right)^{2}+\gamma_{\mathbf{k}_{\|}}^{2} / 4}$,

with $Q_{\mathbf{k}_{\|}}=\Omega_{\mathbf{k}_{\|}} / \gamma_{\mathbf{k}_{\|}}$. In the quasistatic approximation in the "Instantaneous Modes" section, all instantaneous modes present the same width $\gamma_{\mathrm{D}}$, corresponding to the Drude relaxation rate. When retardation becomes significant, we expect the modal widths of retarded plasmon polaritons to be affected; for this reason, in Eq. 35, we have included a mode-dependent width $\gamma_{\mathbf{k}_{\|}}$. A straightforward way to take dissipation effects into account is to solve the characteristic equation with the complex Drude dielectric function $\varepsilon(\omega)=1-\omega_{\mathrm{P}}^{2} /\left[\omega\left(\omega-i \gamma_{\mathrm{D}}\right)\right]$ and a complex frequency $\Omega_{\mathbf{k}_{\|}}=\Omega_{\mathbf{k}_{\|}}^{\prime}+i \Omega_{\mathbf{k}_{\|}}^{\prime \prime}$. To the first order, we find for the imaginary part of $\Omega_{\mathbf{k}_{\|}}$the result

$\Omega_{\mathbf{k}_{\|}}^{\prime \prime}=\frac{\gamma_{\mathbf{k}_{\|}}}{2}=\gamma_{\mathrm{D}} / \sqrt{4+\left(\frac{\omega_{\mathrm{P}}}{c k_{\|}}\right)^{4}}$

An example of the decay rate calculated with Eq. 35 using the effective volumes in Eq. 33 and the modified modal widths in Eq. 36 is represented in Fig. 5 for a dipole located

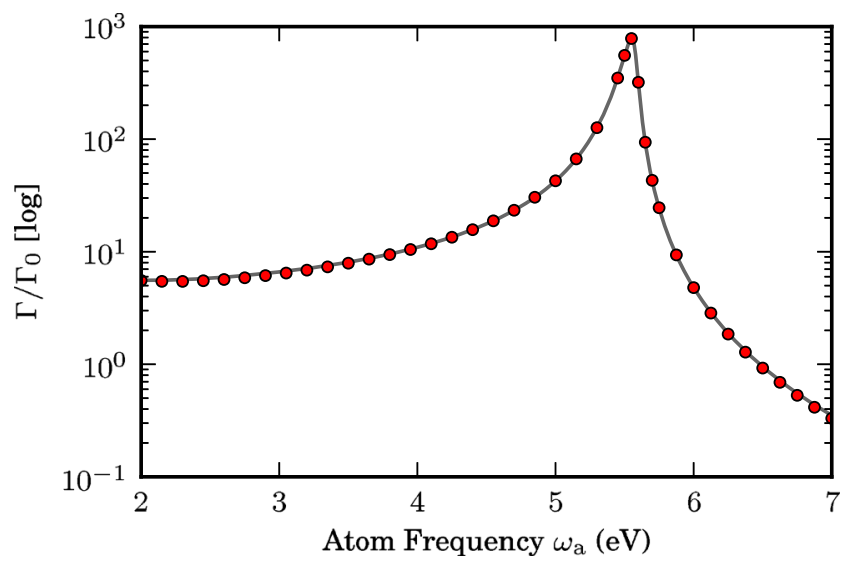

Fig. 5 The normalized decay rate of an atomic dipole directed as $\hat{\mathbf{z}}$ and located $10 \mathrm{~nm}$ above a metal-dielectric interface, as a function of the atomic frequency. The (red) dots are calculated with the Purcell Eq. 35, whereas the solid curve is obtained from the electrodynamical formula in Ref. [2]. The metal-dielectric function follows the Drude model with parameters $\omega_{\mathrm{P}}=7.9 \mathrm{eV}$ and $\gamma_{\mathrm{D}}=60 \mathrm{meV}$ (Ref. [53])

$10 \mathrm{~nm}$ above a silver surface. The agreement with the electrodynamical solution (calculated as in Ref. [2]) is very good, confirming the validity of our formulation. However, the theory presented is not limited to the perturbative approximation, but we believe that it could constitute a useful framework in which to study nonperturbative effects. In particular, the concept of effective volume and its expression in Eq. 33 could be very useful to formulate quantitative estimations in a straightforward way. The quantum formalism is suitable also to the treatment of more recent developments, such as the ultrastrong coupling regime [54-56] or the dynamical Casimir effect [57]. It could also be the starting point for treating nonlinear interaction, similarly to what has been done for dielectric cavities $[58,59]$.

\section{Spherical Geometry}

\section{Radiative Broadening of Plasmons}

In this section, we suppose the region $\mathscr{M}$ to be a spherical particle with radius $R$. The instantaneous plasmons form a discrete spectrum of excitations indexed by the azimuthal quantum number $l$, with frequencies $\omega_{l}=\omega_{\mathrm{P}} \sqrt{l /(2 l+1)}$. The corresponding electrostatic modes (in spherical coordinates) have the following form [31]:

$\mathbf{E}_{\mathrm{qs}, l}(\mathbf{r})=\left\{\begin{array}{r}-\frac{r^{l-1}}{R^{l}}\left[l \mathrm{P}_{l}(\cos \theta) \hat{\mathbf{r}}+\frac{\partial}{\partial \theta} \mathrm{P}_{l}(\cos \theta) \hat{\theta}\right], \\ r<R ; \\ \frac{R^{l+1}}{r^{l+2}}\left[(l+1) \mathrm{P}_{l}(\cos \theta) \hat{\mathbf{r}}-\frac{\partial}{\partial \theta} \mathrm{P}_{l}(\cos \theta) \hat{\theta}\right], \\ r>R ;\end{array}\right.$ 
( $\mathrm{P}_{l}$ indicates the Legendre polynomials). As an effect of the rotational symmetry of the problem, we have considered only $m=0$ modes and dropped the dependence on the azimuth angle $\varphi$. The quasistatic effective volumes calculated from Eq. 11 for a radially oriented atom located outside the particle (at a distance $r_{\mathrm{a}}$ from the center) take the form

$V_{l}\left(r_{\mathrm{a}}, \hat{\mathbf{r}}\right)=\frac{4 \pi r_{\mathrm{a}}^{2 l+4}}{(l+1)^{2} R^{2 l+1}}$,

in agreement with Ref. [60].

The transverse vector potential of Eq. 18 is expanded onto a discretized basis of $N$ vector wavefunctions, as shown in Appendix B. In order to study the effect of retardation, we follow closely the approach in the "General Quantum Formulation" section and look for a family of Hopfield-Bogoljubov operators of the form in Eq. 22. The commutation relation in Eq. 23 can be reduced to the linear eigenproblem

$\mathbb{M}^{(l)} \xi^{(l)}=\left(\frac{\Omega_{l}}{\omega_{\mathrm{P}}}\right)^{2} \xi^{(l)}$

for a $(N+1) \times(N+1)$ matrix $\mathbb{M}^{(l)}$ and a $(N+1)$ dimensional vector $\xi^{(l)}$, defined in Appendix B. When we solve the problem numerically, we obtain a family of $N+$ 1 eigenvalues and eigenvectors [61]. As we have already anticipated, none of the eigenvectors represents of a true stationary state, but they can all be used to extract the density of states according to Eq. 24.

For instance, the density of states of the $l=1$ surface plasmon mode of a $R=1.5 \mathrm{c} / \omega_{\mathrm{P}}$ spherical particle is represented in Fig. 6. The instantaneous mode at $\omega=\omega_{\mathrm{P}} / \sqrt{3}$ (indicated as $\omega_{1}$ ) interacts with the continuum of transverse modes. As a result, the peak acquires a finite linewidth, and its central frequency is redshifted with respect to the instantaneous case. In order to get a quantitative description of the phenomenon, we can extract the central frequency $\Omega_{l}$ of the peak and its full width $\gamma_{\mathrm{rad}, l}$ from the density of states (as shown in Fig. 6) and identify them with the characteristic frequency and the radiative width of the corresponding retarded surface plasmon.

The same procedure is repeated for different radii and azimuthal numbers, leading to the results shown by the dots in Fig. 7. Our data can be compared with the the electrodynamical solutions (solid curves in Fig. 7), calculated as shown at the end of Appendix B. As it can be seen, there is a very good agreement between our results from the density of states and the electrodynamical solutions. In addition, in Fig. $7 \mathrm{~b}$, we have indicated with a dashed curve the value for the radiative width of the $l=1$ mode calculated from

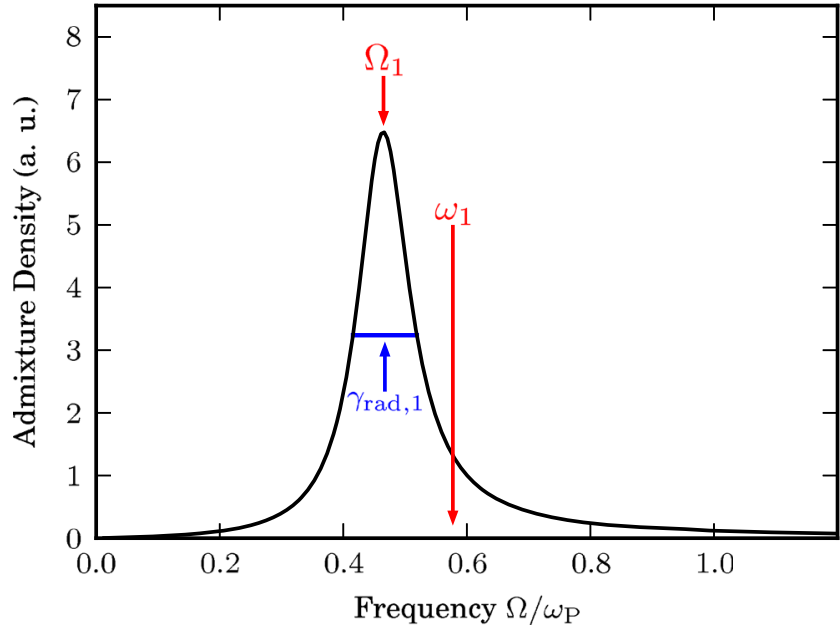

Fig. 6 Density of states (admixture density) of the $l=1$ instantaneous surface plasmon of a $R=1.5 \mathrm{c} / \omega_{\mathrm{P}}$ spherical particle into the continuum of transverse electromagnetic modes. The quantity has been calculated with Eq. 24 from the eigensolutions of Eq. 39. The frequency of the instantaneous plasmon is indicated as $\omega_{1}=\omega_{\mathrm{P}} / \sqrt{3}$. As an effect of retardation, the mode acquires a finite width $\gamma_{\mathrm{rad}, 1}$ and the central frequency is redshifted to $\Omega_{1}$
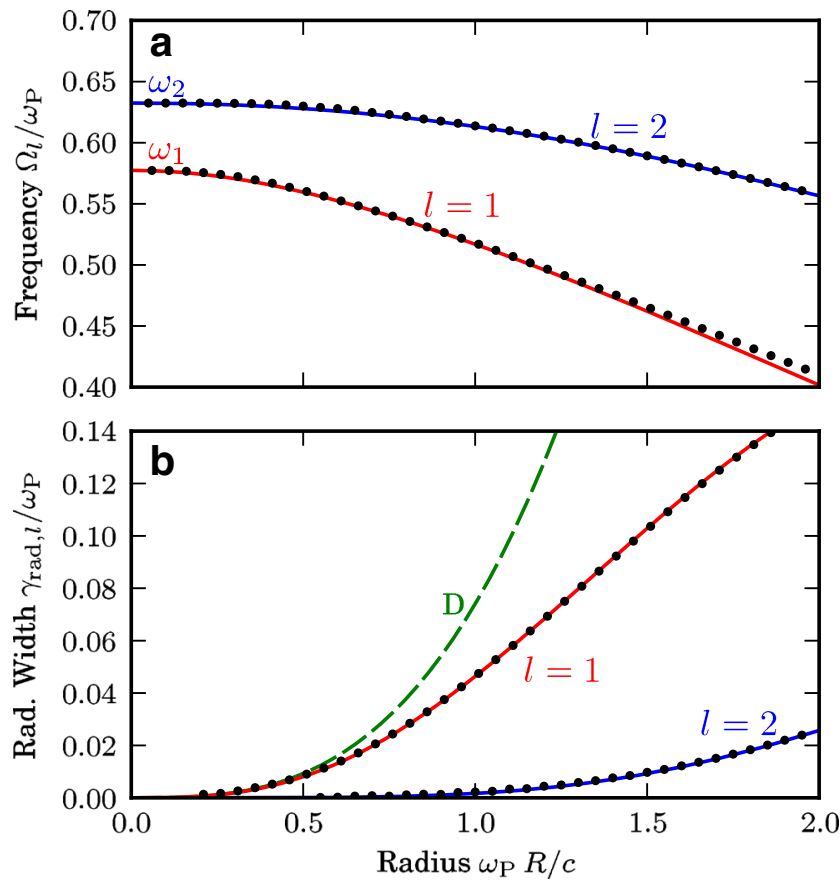

Fig. 7 a Characteristic frequency and $\mathbf{b}$ radiative widths of retarded surface plasmons in a metallic spherical particle, as a function of the sphere radius (normalized to $c / \omega_{\mathrm{P}}$ ) for the azimuthal quantum numbers $l=1,2$. The dots indicate the results obtained by numerically solving the eigenproblem in Eq. 39 and fitting the central frequency and the linewidth onto the admixture density. Solid curves represent the electrodynamical result, calculated as explained in Appendix B. In b, the dashed (green) curve labeled " $\mathrm{D}$ " indicates the dipole approximation of the radiative width in Eq. 40 
the dipolar distribution of the surface charge density in the quasistatic approximation [31]

$\gamma_{\mathrm{rad}, 1}=\frac{2 \omega_{1}}{3}\left(\frac{\omega_{1} R}{c}\right)^{3}$

(with $\omega_{1}=\omega_{\mathrm{P}} / \sqrt{3}$ ). The agreement is good for small radii, whereas the quasistatic value progressively overestimates the correct result with the increasing of $R$. This is mainly due to the increasing difference between the quasistatic frequency $\omega_{1}$ in Eq. 40 and its retarded (and redshifted) counterpart $\Omega_{1}$.

In summary, we believe that the analysis presented in this section could be useful for several reasons. In the first place, we have shown that retardation effects and, in particular, radiative broadening of surface plasmons in metallic nanospheres could be understood in the context of the Fano-Anderson theory of a discrete quantum state (the instantaneous plasmon) in resonance with the continuum of transverse electromagnetic modes. The results obtained with this theory are in both qualitative and quantitative agreement with electrodynamical calculations. In the second place, we believe that our results could contribute to clarifying the limits of validity of the electrostatic approach, which is often employed for the study of confined surface plasmons, especially in the case of more complex geometries.

\section{Interaction with Matter}

When we put a radially oriented atom at $r_{\mathrm{a}}$, as an effect of retardation, the decay rate is modified with respect to the quasistatic situation. The modification can be readily evaluated with our formalism, since retarded surface plasmons, being resonances with a finite linewidth, provide a continuous density of final states to which the Fermi golden rule can be applied. Each eigenvector of Eq. 39 (identified by the index $j$ ) defines the quantum operator $\alpha_{l}^{(j)}$ (see Eq. 56 in Appendix B) and, consequently, can be related to an electromagnetic field mode through the relation $\mathbf{E}_{l}^{(j)}=$ $\left[\alpha_{l}^{(j)}, \mathrm{E}_{\mathrm{qs}}-\dot{\mathbf{A}}\right]$. With a procedure similar to that leading to Eq. 52, we obtain

$$
\begin{aligned}
& \mathbf{E}_{l}^{(j)}(\mathbf{r})=\left(W^{(j)}-Y^{(j)}\right) \sqrt{\frac{\hbar \omega_{l}}{8 \pi \varepsilon_{0} R}} \mathbf{E}_{\mathrm{qs}, l}(\mathbf{r})- \\
& i \sum_{v} c k_{v}\left[X_{v}^{(j)}+Z_{v}^{(j)}\right] \mathbf{A}_{\mathrm{T}, l, k_{v}}(\mathbf{r}) .
\end{aligned}
$$

The contribution of the $l^{\text {th }}$ retarded mode to the decay rate can be calculated by applying directly the Fermi golden rule

$\Gamma_{l}=\sum_{j} \frac{2 \pi}{\hbar^{2}}\left|\mu \cdot \mathbf{E}_{l}^{(j)}\left(\mathbf{r}_{\mathrm{a}}\right)\right|^{2} \delta\left(\Omega_{l}^{(j)}-\omega_{\mathrm{a}}\right)$, where $\Omega_{l}^{(j)}$ is the corresponding eigenvalue of Eq. 39. The total decay is obtained by summing over all azimuthal numbers: $\Gamma=\sum_{l} \Gamma_{l}$. As an example, in Fig. 8, the dots indicated the $l=1$ contribution to the decay rate of an atom located in proximity of a $R=1.5 c / \omega_{\mathrm{P}}$ spherical particle, calculated with Eq. 42. Our data are shown to be in very good agreement with the electrodynamical result calculated with the Mie theory [4-6]. In particular, we stress that the decay rate is characterized by an asymmetric lineshape that resembles a Fano resonance, as it has been already observed $[62,63]$. When the particle radius is reduced and the quasistatic limit is approached, the decay rate lineshape progressively transforms into a symmetric Lorentzian, since instantaneous plasmons are true localized states.

In our treatment of spherical particles, we have neglected the effect of light dissipation inside the metal. In applying our results to real systems, dissipation should be taken into account, since it is responsible of an additional broadening of the plasmonic resonances. A minimal approach to include dissipation effects is to define a total plasmon modal width $\gamma_{l}=\gamma_{\mathrm{rad}, l}+\gamma_{\mathrm{D}}$, including both radiative and nonradiative broadening. The nonradiative width can be approximated with the damping constant $\gamma_{D}$ of the Drude model. More elaborated models involve solving Eq. 57 with a complex dielectric function and extracting the modal width from the imaginary part of the solution.

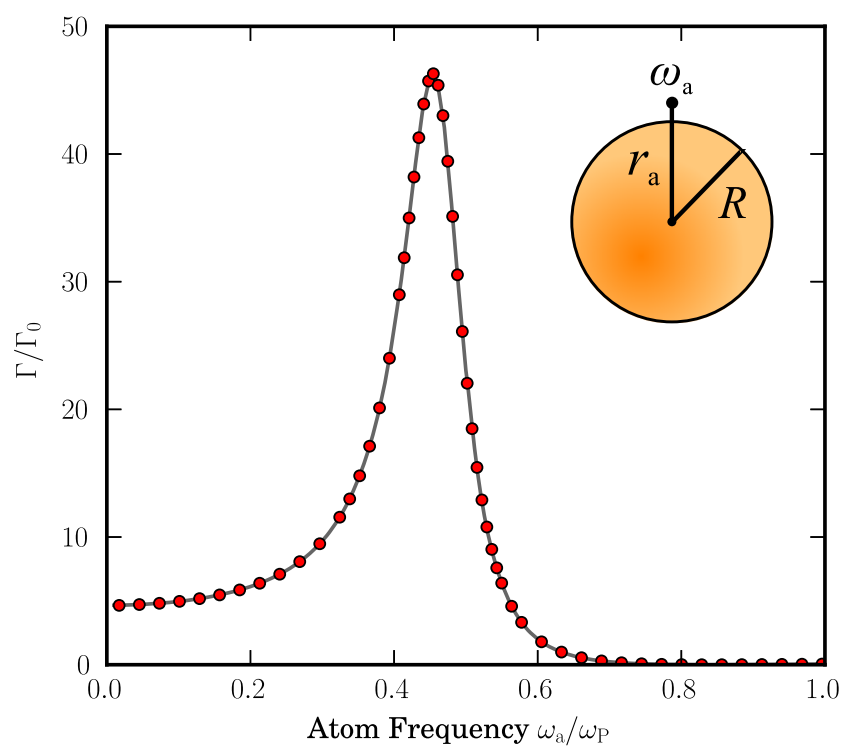

Fig. 8 The $l=1$ contribution to the normalized decay rate of an atomic dipole moment located in proximity to $R=1.5 c / \omega_{\mathrm{P}}$ metallic spherical particle. The dipole is radially oriented and at a distance $r_{\mathrm{a}}=1.2 R$ from the center of the sphere (see inset). The (red) dots are calculated with Fermi golden rule according to Eq. 42, whereas the solid curve is obtained from classical electrodynamics (Mie theory) 
The agreement with the Mie theory confirms the validity of the retarded formalism in the perturbative approximation. As for the case of the planar surface, however, we believe that the quantum model presented in this work could be used beyond the limits of the perturbation theory, to study nonperturbative, nonlinear, or even nonclassical effects. Moreover, we stress that the numerical procedure reported here for metallic nanospheres could be generalized to arbitrary geometries, including nanoparticles of more complex shape, which are commonly investigated in plasmonics [64].

\section{Conclusions}

In this work, we establish a link between the theory of exciton-polaritons and that of retarded surface plasmons. Quantized instantaneous surface plasmons play the role of the exciton and interact with the continuum of electromagnetic transverse modes. A new family of bosonic quantum operators, which are to be associated to retarded surface plasmons, is obtained with the help of a Hopfield-Bogoljubov transformation. The interaction between plasmons and nearby dipolar emitters is treated with a cavity QED-inspired formalism based on the concept of effective volume. As examples of application, the general theory is worked out for the cases of a planar vacuum-metal interface and a spherical metallic nanoparticle.

We have considered the ideal case of a free electron gas without dissipation, because we are interested in highlighting the modification of the dispersive properties of plasmonic modes as an effect of retardation, e.g., the phenomenon of radiative broadening of retarded plasmons in confined nanoparticles. Nevertheless, we believe that our results can be applied even to less ideal materials. In particular, we have often indicated how to extend the formalism in order to include the effect of a finite linewidth for instantaneous modes and the relevant equations, such as the definition of the effective volume (Eq. 11), are presented in a form suitable for an arbitrary metal-dielectric function.

The formalism presented is not limited to the study of plasmon-matter interaction in perturbation theory, but it constitutes a full quantum framework analogous to those widely available in the context of cavity QED. We believe that it can represent an interesting basis for further theoretical developments, such as the study of nonperturbative, nonlinear, nonclassical, or vacuum-related quantum effects. Such effects, which are currently of great interest for exciton-polaritons and for intersubband polaritons, could find a viable experimental platform within the emerging area of quantum plasmonics.

\section{Appendix A: Planar Geometry}

In this appendix, we sketch the derivation of the retarded plasmon polariton modes with the help of a HopfieldBogoljubov transformation of the instantaneous plasmons coupled to the transverse electromagnetic field.

The interaction terms of the Hamiltonian in Eq. 14 assume the form

$$
\begin{aligned}
H_{\mathrm{I}} & =i \int \mathrm{d}^{2} \mathbf{k}_{\|} \mathrm{d} k_{z} \hbar C\left(k_{z} ; \mathbf{k}_{\|}\right)\left(\mathrm{b}_{\mathbf{k}_{\|}^{\dagger}}^{\dagger}-\mathrm{b}_{-\mathbf{k}_{\|}}\right)\left(\mathrm{a}_{\mathbf{k}_{\|}, k_{z}}+\mathrm{a}_{-\mathbf{k}_{\|},-k_{z}}^{\dagger}\right), \\
H_{\mathrm{II}} & =\int \mathrm{d}^{2} \mathbf{k}_{\|} \mathrm{d} k_{z} \mathrm{~d} k_{z}^{\prime} \hbar D\left(k_{z}, k_{z}^{\prime} ; \mathbf{k}_{\|}\right)\left(\mathrm{a}_{\mathbf{k}_{\|}, k_{z}}+\mathrm{a}_{-\mathbf{k}_{\|},-k_{z}}^{\dagger}\right) \\
& \times\left(\mathrm{a}_{\mathbf{k}_{\|}, k_{z}^{\prime}}^{\dagger}+\mathrm{a}_{-\mathbf{k}_{\|},-k_{z}^{\prime}}\right),
\end{aligned}
$$

with the coefficients

$$
\begin{aligned}
& C\left(k_{z} ; \mathbf{k}_{\|}\right)=\frac{\omega_{\mathrm{P}}^{2}}{4 k} \sqrt{\frac{k_{\|}}{\pi c k \omega_{\mathrm{s}}}} ; \quad \tilde{D}\left(k_{z}, k_{z}^{\prime} ; \mathbf{k}_{\|}\right)=\frac{i \omega_{\mathrm{P}}^{2}\left(k_{z} k_{z}^{\prime}+k_{\|}^{2}\right)}{8 c \pi\left(k k^{\prime}\right)^{\frac{3}{2}}} \\
& D\left(k_{z}, k_{z}^{\prime} ; \mathbf{k}_{\|}\right)=\frac{\omega_{\mathrm{P}}^{2}}{8 c k} \delta\left(k_{z}-k_{z}^{\prime}\right)+\mathscr{P} \frac{\tilde{D}\left(k_{z}, k_{z}^{\prime} ; \mathbf{k}_{\|}\right)}{k_{z}^{\prime}-k_{z}}
\end{aligned}
$$

( $\mathscr{P}$ denotes the principal value).

The diagonalization of the Hamiltonian in Eq. 14 is obtained by replacing the expansion of Eq. 29 into Eq. 23 and collecting the terms in front of the unperturbed operators $b_{\mathbf{k}_{\|}}, b_{-\mathbf{k}_{\|}}^{\dagger}, \mathrm{a}_{\mathbf{k}_{\|}, k_{z}}$, and $\mathrm{a}_{-\mathbf{k}_{\|},-k_{z}}^{\dagger}$, so that we obtain a system of four equations for the coefficients $W, X\left(k_{z}\right), Y, Z\left(k_{z}\right)$. With some algebraic manipulation, the equations can be condensed into the form

$$
\begin{aligned}
& \frac{c}{2 k}\left(k_{z}^{2}+\mathbf{k}_{\|}^{2}-\frac{\Omega^{2}}{c^{2}}+\frac{\omega_{\mathrm{P}}^{2}}{2 c^{2}}\right) \xi\left(k_{z}\right) \\
& \quad+\mathscr{P} \int \mathrm{d} k_{z}^{\prime} 2 \frac{\tilde{D}\left(k_{z}, k_{z}^{\prime}\right)}{k_{z}^{\prime}-k_{z}} \xi\left(k_{z}^{\prime}\right)=-i(W+Y) C(k),
\end{aligned}
$$

where $\xi\left(k_{z}\right)=X\left(k_{z}\right)-Z\left(k_{z}\right)$.

Equation 45 can be solved with Kramers-Kronig relations, but attention must be paid to the fact that the integrand (considered as a function of the complex variable $k_{z}^{\prime}$ ) has additional poles in addition to that at $k_{z}^{\prime}=k_{z}$. In particular, if we introduce the quantities

$$
\Delta_{-}^{2}=\mathbf{k}_{\|}^{2}-\frac{\Omega^{2}}{c^{2}}+\frac{\omega_{\mathrm{P}}^{2}}{c^{2}}, \quad \Delta_{+}^{2}=\mathbf{k}_{\|}^{2}-\frac{\Omega^{2}}{c^{2}},
$$

and write $\xi\left(k_{z}\right)$ in the form ( $\mathscr{C}$ is an arbitrary constant)

$$
\xi\left(k_{z}\right)=\frac{i \mathscr{C}}{\sqrt{k}\left(k_{z}+i \Delta_{+}\right)\left(k_{z}-i \Delta_{-}\right)},
$$


upon integrating the integrand on a closed circuit in the upper complex plane like the one in Fig. 9 and taking the limit $\left|k_{z}^{\prime}\right| \rightarrow \infty$, the application of the Cauchy theorem leads to the expression

$$
\begin{aligned}
& \frac{c}{2 k^{\frac{3}{2}}}\left\{\left[\frac{i\left(\Delta_{+}^{2}+k_{z}^{2}\right)\left(\Delta_{-}^{2}-k_{\|}^{2}\right)-\left(i k_{z} \Delta_{-}+k_{\|}^{2}\right)\left(k_{z}+i \Delta_{+}\right)\left(\Delta_{-}-\Delta_{+}\right)}{\left(k_{z}+i \Delta_{+}\right)\left(k_{z}-i \Delta_{-}\right)\left(\Delta_{-}^{2}-k_{\|}^{2}\right)}\right]\right. \\
& \left.+\frac{i \omega_{\mathrm{P}}^{2}}{2 c^{2}\left(k_{\|}+\Delta_{+}\right)\left(\Delta_{-}-k_{\|}\right)}\right\} \mathscr{C}=-i(W+Y) C(k) .
\end{aligned}
$$

The equation has a nonzero solution for the constant $\mathscr{C}$ if and only if the $\left(k_{z}\right.$-dependent) term in the square brackets cancels; this happens when $\Delta_{+} \Delta_{-}=k_{\|}^{2}$, i.e.,

$\Omega_{\mathbf{k}_{\|}}^{4}-\Omega_{\mathbf{k}_{\|}}^{2}\left[2\left(c k_{\|}\right)^{2}+\omega_{\mathrm{P}}^{2}\right]+\left(c k_{\|} \omega_{\mathrm{P}}\right)^{2}=0$,

whence the plasmon polariton dispersion relation in Eq. 30 is recovered.

The constant $\mathscr{C}$ takes the value

$\mathscr{C}=-k_{\|} \sqrt{\frac{c k_{\|}}{\pi \omega_{\mathrm{s}}}}\left(\Delta_{-}-\Delta_{+}\right)(W+Y)$.

Once $\mathscr{C}$ is known, from the original system of equations, it is possible to work out the expression for the coefficients in the expansion of $\alpha_{\mathbf{k}_{\|}}$:

$$
\begin{aligned}
& \xi\left(k_{z}\right)=-i k_{\|} \sqrt{\frac{c k_{\|}}{\pi k \omega_{\mathrm{s}}}} \frac{\Delta_{-}-\Delta_{+}}{\left(k_{z}+i \Delta_{+}\right)\left(k_{z}-i \Delta_{-}\right)} ; \\
& W=\mathscr{K}\left(1+\frac{\Omega_{\mathbf{k}_{\|}}}{\omega_{\mathrm{s}}}\right) ; \quad Y=\mathscr{K}\left(1-\frac{\Omega_{\mathbf{k}_{\|}}}{\omega_{\mathrm{s}}}\right) ; \\
& X=\mathscr{K}\left(1+\frac{\Omega_{\mathbf{k}_{\|}}}{c k}\right) \xi ; \quad Z=\mathscr{K}\left(\frac{\Omega_{\mathbf{k}_{\|}}}{c k}-1\right) \xi .
\end{aligned}
$$

The arbitrary constant $\mathscr{K}$ can be fixed from the normalization conditions $\left\langle\Psi\left|\alpha_{\mathbf{k}_{\|}} \alpha_{\mathbf{k}_{\|}}^{\dagger}-\alpha_{\mathbf{k}_{\|}}^{\dagger} \alpha_{\mathbf{k}_{\|}}\right| \Psi\right\rangle=1$ on a generic normalized quantum state $|\Psi\rangle$. The result is

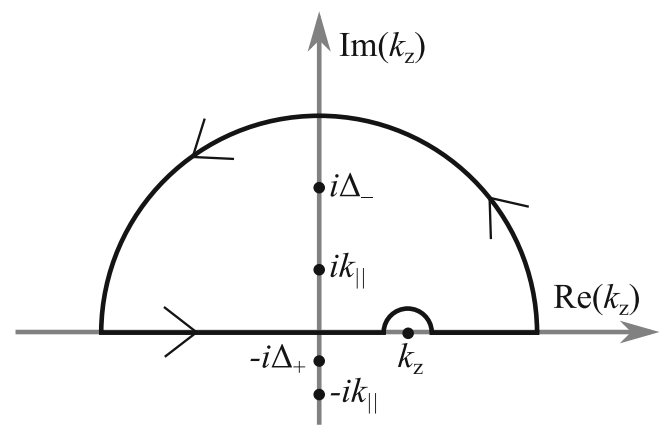

Fig. 9 The path of integration of Eq. 45 in the complex $k_{z}$ plane
$\mathscr{K}^{2}=\frac{1}{4}\left(\frac{\omega_{\mathrm{s}}}{\Omega_{\mathbf{k}_{\|}}}\right) \frac{\left(\Delta_{-}+\Delta_{+}\right) k_{\|}}{\Delta_{-}^{2}+\Delta_{+}^{2}}$.

The behavior of $W$ and $Y$ as a function of $k_{\|}$is reported in Fig. 1.

The electric field operator is expanded onto a family of modes $\mathbf{E}_{\mathbf{k}_{\|}}$(r) according to Eq. 32. The expression for the modes can be calculated from the commutation relation $\left[\alpha_{\mathbf{k}_{\|}}, \mathrm{E}_{\mathrm{qs}}-\dot{\mathrm{A}}\right]=\mathbf{E}_{-\mathbf{k}_{\|}}$, which leads to

$$
\begin{aligned}
\mathbf{E}_{\mathbf{k}_{\|}}(\mathbf{r})= & (W-Y) \sqrt{\frac{\hbar \omega_{\mathrm{s}}}{16 \pi^{2} \varepsilon_{0} k_{\|}}} \mathbf{E}_{\mathrm{qs}, \mathbf{k}_{\|}}(\mathbf{r})- \\
& i \int \mathrm{d} k_{z} c k\left[X\left(-k_{z}\right)+Z\left(-k_{z}\right)\right] \mathbf{A}_{\mathrm{T}, \mathbf{k}_{\|}, k_{z}}(\mathbf{r})
\end{aligned}
$$

$\left(k=\sqrt{k_{\|}^{2}+k_{z}^{2}}\right)$. With the help of Eq. 50 and some results on Fourier transforms in the $k_{z}$ space, we find

$\mathbf{E}_{\mathbf{k}_{\|}}=\mathscr{K} \frac{\Omega_{\mathbf{k}_{\|}} k_{\|}}{\pi\left(\Delta_{+}+\Delta_{-}\right)} \sqrt{\frac{\hbar k_{\|}}{\varepsilon_{0} \omega_{\mathrm{s}}}}\left(-i \hat{\mathbf{k}_{\|}} \pm \frac{k_{\|}}{\Delta_{ \pm}} \hat{\mathbf{z}}\right) e^{i \mathbf{k}_{\|} \cdot \rho \mp \Delta_{ \pm} z}$

(the upper and lower signs refer to the regions $z>0$ and $z<0$, respectively, $\hat{\mathbf{k}_{\|}}$is the unit vector directed as $\left.\mathbf{k}_{\|}\right)$. Equation 53 is analogous to the expression of the field obtained from classical electrodynamics [3, 21].

\section{Appendix B: Spherical Geometry}

In this appendix, we briefly present how the eigenvalue Eq. 39 is derived. The transverse vector potential of Eq. 18 is expanded onto the electromagnetic vector wave functions [65]

$$
\begin{aligned}
\mathbf{A}_{\mathrm{T}, l, k}^{(1)}(\mathbf{r})=-\mathscr{N} j_{l} k r \frac{\partial}{\partial \theta} \mathrm{P}_{l}(\cos \theta) \hat{\varphi} & \\
\mathbf{A}_{\mathrm{T}, l, k}^{(2)}(\mathbf{r})=\mathscr{N}[ & {\left[l(l+1) \frac{j_{l}(k r)}{k r} \mathrm{P}_{l}(\cos \theta) \hat{\mathbf{r}}\right.} \\
& \left.+\frac{1}{k r} \frac{\partial}{\partial r}\left[r j_{l}(k r)\right] \frac{\partial}{\partial \theta} \mathrm{P}_{l}(\cos \theta) \hat{\theta}\right]
\end{aligned}
$$


$\left(j_{l}(x)\right.$ denotes the spherical Bessel function of order $l$ ). Polarizations with $\lambda=1$ and 2 correspond to TE and TM modes, respectively. ${ }^{3}$ Transverse modes are indexed by the continuous wavenumber $k$, which is related to the modal frequency $\omega_{k}=c k$. For each $l$, the frequencies $\omega_{k}$ encompass the whole spectrum, overlapping with the instantaneous frequencies $\omega_{l}$.

The continuous index $k$ is discretized into a finite number of retarded modes up to a cutoff value $N$ (the modes are labeled by the index $v=1,2, \ldots, N)$. This can be accomplished by supposing to enclose the system in a large sphere of radius $L$, with $L>>R$. According to the SturmLiouville theory [66], the values $k_{v}$ implicitly provided by the equation

$j_{l}\left(k_{v} L\right)=0$,

constitute an orthogonal basis of electromagnetic modes. Consequently, the normalization factor $\mathscr{N}$ can be shown to assume the form

$\mathscr{N}=\sqrt{\frac{\hbar(2 l+1)}{4 \pi l(l+1) \varepsilon_{0} c k_{v} L^{3} j_{l+1}^{2}\left(k_{v} L\right)}}$.

We look for Hopfield-Bogoljubov operators of the form

$\alpha_{l}=W_{l} \mathrm{~b}_{l}+Y_{l} \mathrm{~b}_{l}^{\dagger}+\sum_{v}\left(X_{l, v} \mathrm{a}_{v}+Z_{l, v} \mathrm{a}_{v}^{\dagger}\right)$,

which satisfy the commutation relation in Eq. 23. After defining the adimensional quantities $\tilde{\omega}_{l}=\omega_{l} / \omega_{\mathrm{P}}, \tilde{k}=$ $c k / \omega_{\mathrm{P}}, \tilde{R}=\omega_{\mathrm{P}} R / c$, and $\tilde{L}=\omega_{\mathrm{P}} L / c$, we can introduce the $(\mathrm{N}+1)$-dimensional vector $\xi^{(l)}$, whose components are $(\mathscr{K}$ is a normalization constant):

$\xi_{v}^{(l)}=-i \frac{\mathscr{K}}{\sqrt{\tilde{k}}}\left(X_{l, v}-Z_{l, v}\right), \quad v=1, \ldots, N$;

$\xi_{N+1}^{(l)}=\frac{\mathscr{K}}{\sqrt{\tilde{\omega}_{l}}}\left(W_{l}+Y_{l}\right)$

By collecting the terms in front of the quantum operators $\mathrm{b}_{l}, \mathrm{~b}_{l}^{\dagger}, \mathrm{a}_{l, v}$, and $\mathrm{a}_{l, v}^{\dagger}$, Eq. 23 can be reduced to a linear system of equations. With some further algebraic manipulation, we are led to the eigenproblem in Eq. 39, with

${ }^{3}$ Notice that in Ref. [65] a different notation is used. Polarizations with $\lambda=1$ and 2 represent $\mathbf{M}$ and $\mathbf{N}$ vector wave functions, respectively. the $(N+1) \times(N+1)$ symmetric matrix $\mathbb{M}^{(l)}$ defined as follows:

$$
\begin{aligned}
\mathbb{M}_{v, v}^{(l)}=\frac{\tilde{R}^{3}}{j_{l+1}^{2}\left(k_{v} L\right) \tilde{L}^{3}}[ & j_{l+1}^{2}\left(k_{v} R\right)+j_{l}^{2}\left(k_{v} R\right)\left(1+\frac{2(l+1)}{\left(k_{v} R\right)^{2}}\right) \\
& \left.-(2 l+3) \frac{j_{l}\left(k_{v} R\right) j_{l+1}\left(k_{v} R\right)}{k_{v} R}\right]+\tilde{k}_{v}^{2} ;
\end{aligned}
$$

$\mathbb{M}_{\nu, \mu}^{(l)}=\frac{2 \tilde{R}}{\left|j_{l+1}\left(k_{\nu} L\right) j_{l+1}\left(k_{\mu} L\right)\right| \tilde{L}^{3}}$

$\times\left\{\frac{\tilde{R}}{\tilde{k}_{\nu}^{2}-\tilde{k}_{\mu}^{2}}\left[\tilde{k}_{\mu} j_{l}\left(k_{\mu} R\right) j_{l+1}\left(k_{\nu} R\right)-\tilde{k}_{\nu} j_{l}\left(k_{\nu} R\right) j_{l+1}\left(k_{\mu} R\right)\right]\right.$

$\left.+\frac{l+1}{\tilde{k}_{\nu} \tilde{k}_{\mu}} j_{l}\left(k_{\nu} R\right) j_{l}\left(k_{\mu} R\right)\right\}$;

$\mathbb{M}_{\nu, N+1}^{(l)}=\mathbb{M}_{N+1, \nu}^{(l)}=-\sqrt{\frac{2 l(l+1)}{(2 l+1) \tilde{R} \tilde{L}^{3}}} \frac{\tilde{R} j_{l}\left(k_{\nu} R\right)}{\tilde{k}_{\nu}\left|j_{l+1}\left(k_{\nu} L\right)\right|} ;$

$\mathbb{M}_{N+1, N+1}^{(l)}=\tilde{w}_{l}^{2} ; \quad$ with $\quad v, \mu=1,2, \ldots, N \quad$ and $\quad v \neq \mu$.

The eigenproblem is solved with numerical methods. The value of the coefficients $W_{l}, Y_{l}, X_{l, v}$, and $Z_{l, v}$ can be extracted from the definition of eigenvector $\xi^{(l)}$ with the additional relations

$\omega_{l}\left(W_{l}-Y_{l}\right)=\Omega_{l}\left(W_{l}+Y_{l}\right) ;$

$c k\left(X_{l, v}+Z_{l, v}\right)=\Omega_{l}\left(X_{l, v}-Z_{l, v}\right)$.

The normalization constant can be calculated from the condition $\left\langle\Psi\left|\alpha_{l} \alpha_{l}^{\dagger}-\alpha_{l}^{\dagger} \alpha_{l}\right| \Psi\right\rangle=1$ with the operator in Eq. 56. The result is $\left|\xi^{(l)}\right|^{2}=\omega_{\mathrm{P}} / \Omega_{l}$, which, for an eigenvector with unitary normalization, leads simply to $\mathscr{K}=\sqrt{\omega_{\mathrm{P}} / \Omega_{l}}$.

The solutions of the vector eigenproblem (39) are shown by the dots in Fig. 7 and compared with the corresponding electrodynamical solutions. The latter are obtained by solving for a complex $\omega$ the equation

$\frac{h_{l}^{(1)}(k R)}{\frac{\partial}{\partial r}\left[r h_{l}^{(1)}(k r)\right]_{r=R}}=\varepsilon(\omega) \frac{i_{l}(n k R)}{\frac{\partial}{\partial r}\left[r i_{l}(n k r)\right]_{r=R}}$,

with a Drude dielectric function $\varepsilon=1-\omega_{\mathrm{P}}^{2} / \omega^{2}$. In the equation, $k=\omega / c, n=\sqrt{\varepsilon}, h_{l}^{(1)}(x)$ is the spherical Hankel function of the first kind, and $i_{l}(x)$ is the modified spherical Bessel function of the first kind. The modal frequency and radiative width are related to the real and imaginary parts of the solution by $\Omega_{l}=\Re(\omega)$ and $\gamma_{\mathrm{rad}}=-2 \Im(\omega)$, respectively.

\section{References}

1. Stockman MI (2011) Opt Express 19(22):22029

2. Ford G, Weber W (1984) Phys Rep 113(4):195 
3. Raether H (1988) Surface plasmons on smooth and rough surfaces and on gratings. Springer tracts in modern physics, vol 111. Springer, Berlin

4. Kim YS, Leung P, George TF (1988) Surf Sci 195:1

5. Dung HT, Knöll L, Welsch DG (2001) Phys Rev A 64:013804

6. Mertens H, Koenderink AF, Polman A (2007) Phys Rev B 76:115123

7. Mayergoyz ID, Fredkin DR, Zhang Z (2005) Phys Rev B 72:155412

8. Hohenester U, Krenn J (2005) Phys Rev B 72:195429

9. Hohenester U, Trügler A (2012) Comput Phys Commun 183(2):370

10. Yurkin MA, Hoekstra AG (2011) Quant J Spectrosc Ra 112(13):2234

11. D'Agostino S, Della Sala F, Andreani LC (2013) Phys Rev B 87:205413

12. Mohammadi A, Sandoghdar V, Agio M (2008) New J Phys 10(10): 105015

13. Huttner B, Baumberg JJ, Barnett SM (1991) Europhys Lett 16(2): 177

14. Dung HT, Knöll L, Welsch DG (1998) Phys Rev A 57:3931

15. Di Stefano O, Savasta S, Girlanda R (2000) Phys. Rev. A 61:023803

16. Bhat NAR, Sipe JE (2006) Phys Rev A 73(6):063808

17. Van Vlack C, Kristensen PT, Hughes S (2012) Phys. Rev. B 85(7):075303

18. D'Agostino S, Alpeggiani F, Andreani LC (2013) Opt Express 21:27602

19. Ge RC, Van Vlack C, Yao P, Young JF, Hughes S (2013) Phys Rev B 87(20):205425

20. Ritchie RH, Wilems RE (1969) Phys Rev 178:372

21. Elson JM, Ritchie RH (1971) Phys Rev B 4:4129

22. Babiker M, Barton G (1976) Phys J A Math Gen 9(1):129

23. Barton G (1979) Rep Prog Phys 42(6):963

24. Ballester D, Tame MS, Lee C, Lee J, Kim MS (2009) Phys Rev A 79:053845

25. Archambault A, Marquier F, Greffet JJ, Arnold C (2010) Phys Rev B 82:035411

26. Bergman DJ, Stockman MI (2003) Phys Rev Lett 90:027402

27. Gerard JM, Gayral B (1999) J Lightwave Technol 17(11):2089

28. Kristensen PT, Van Vlack C, Hughes S (2012) Opt Lett 37(10): 1649

29. Sauvan C, Hugonin JP, Maksymov IS, Lalanne P (2013) Phys Rev Lett 110(23):237401

30. Maier SA (2006) Opt Express 14(5):1957

31. Sun G, Khurgin JB (2011) In: Shvets G, Tsukerman I (eds) Plasmonics and plasmonic metamaterials: analysis and applications. World Scientific, Singapore

32. Agio M (2012) Nanoscale 4:692

33. Fano U (1956) Phys Rev 103:1202

34. Hopfield JJ (1958) Phys Rev 112:1555

35. Agranovich V (1960) Sov Phys JETP 37:307
36. Andreani LC (1995) In: Burstein E, Weisbuch C (eds) Confined electrons and photons, NATO ASI series, vol 340. Springer, Berlin, pp 57-112

37. Bogoljubov N (1958) Il Nuovo Cimento 7:794

38. Quattropani A, Andreani LC, Bassani F (1986) Il Nuovo Cimento D 7(1):55

39. Brako R, Hrnčević J, Šunjić M (1975) Z Phys B 21:193

40. Landau L, Lifshitz E, Pitaevskii L (1984) Electrodynamics of continuous media. Course of theoretical physics, vol 8, 2nd edn. Pergamon, Oxford

41. Larkin IA, Stockman MI, Achermann M, Klimov VI (2004) Phys Rev B 69:121403

42. Scholl JA, Koh AL, Dionne JA (2012) Nature 483(7390):421

43. Trügler A, Hohenester U (2008) Phys Rev B 77(11):115403

44. Waks E, Sridharan D (2010) Phys Rev A 82(4):043845

45. Savasta S, Saija R, Ridolfo A, Di Stefano O, Denti P, Borghese F (2010) ACS Nano 4(11):6369

46. He Y, Jiang C, Chen B, Li JJ, Zhu KD (2012) Opt Lett 37(14):2943

47. Dvoynenko MM, Wang JK (2013) Opt Lett 38(5):760

48. Andreani LC, Panzarini G, Gérard JM (1999) Phys Rev B 60:13276

49. Alpeggiani F, D’Agostino S, Andreani LC (2012) Phys Rev B 86:035421

50. Todorov Y, Sirtori C (2012) Phys Rev B 85:045304

51. Anderson PW (1961) Phys Rev 124:41

52. Fano U (1961) Phys Rev 124:1866

53. Barnes WL (2006) J Opt A Pure Appl Opt 8(4):S87

54. Ciuti C, Bastard G, Carusotto I (2005) Phys Rev B 72:115303

55. Ciuti C, Carusotto I (2006) Phys Rev A 74:033811

56. Stassi R, Ridolfo A, Di Stefano O, Hartmann MJ, Savasta S (2013) Phys Rev Lett 110:243601

57. Dodonov V (2010) Phys Scr 82(3):038105

58. Ferretti S, Gerace D (2012) Phys Rev B 85:033303

59. Ferretti S, Savona V, Gerace D (2013) New J Phys 15(2):025012

60. Colas des Francs G, Derom S, Vincent R, Bouhelier A, Dereux A (2012) Int J Opt 2012:1

61. Gerace D, Andreani LC (2007) Phys Rev B 75:235325

62. Luk'yanchuk B, Zheludev NI, Maier SA, Halas NJ, Nordlander P, Giessen H, Chong CT (2010) Nat Mater 9(9):707

63. Giannini V, Francescato Y, Amrania H, Phillips CC, Maier SA (2011) Nano Lett 11(7):2835

64. D'Agostino S (2013) In: Della Sala F, D'Agostino S (eds) Handbook of molecular plasmonics, chap 3. Pan Stanford Publishing, Singapore, p 137

65. Tai CT (1993) Dyadic Green functions in electromagnetic theory. IEEE, Piscataway

66. Prosperetti A (2011) Advanced mathematics for applications. Cambridge University Press, Cambridge 